\title{
Optimal Extraction of Photovoltaic Cell Parameters for the Maximization of Photovoltaic Power Output Using a Hybrid Particle Swarm Grey Wolf Optimization Algorithm
}

\author{
*Author: Ali Abubakar \\ Department of Mathematics, Kwame Nkrumah University of Science and Technology, Ghana \\ Author: Dr. Reindorf Nartey Borkor \\ Department of Mathematics, Kwame Nkrumah University of Science and Technology, Ghana \\ Email: a.abubakar7751@gmail.com * \\ Email: reinbork@gmail.com
}

\begin{abstract}
Avoiding over-dependency on the oil-fired energy supply systems motivates many countries to integrate renewable energy into the existing energy supply systems. Solar Photovoltaic technology forms the most promising option for developing such a costeffective and sustainable energy supply system. Generally, the current-voltage curve is used in the performance assessment and analysis of the Photovoltaic module. The accuracy of the equations for the curve depends on accurate cell parameters. However, the extraction of these parameters remains a complex stochastic nonlinear optimization problem. Many studies have been carried out to deal with such problem but still more researches need to be carried out to achieve a minimum error and a high accuracy. The existing researches ignored the variation in the meteorological data though it has a significant impact on the problem design. In this study, the Sample Average Approximation was employed to deal with the uncertainty and the hybrid optimization method was used to get the optimal parameters. The results showed that the Hybrid PSO-GWO produced the most optimal solution: Series resistance(1.4623), Shunt resistance $(215.0000)$, Ideal diode factors $\left(n_{1}=0.9500, n_{2}=1.6500\right)$ with a maximum PV
\end{abstract}


Academic Journal of Research and Scientific Publishing | Vol 3 | Issue 28

Publication Date: 5-8-2021 ISSN: 2706-6495

power of $59.850 \mathrm{~W}$. The methodology produced realistic results since the variability is dealt with and the Hybrid PSO-GWO finds the optimal solution at a higher convergence rate.

Keywords: Diode, Irradiance, Monte-Carlo, Parameters, Photovoltaic, Solar, Stochastic

\section{Introduction}

Globally, the greater portion of the total energy supply is from the oil-fired energy resources (Kamjoo, 2015). However, there are series of negative effects associated with the use of these conventional resources. Some of these effects are the emission of $\mathrm{CO}_{2}$ which could aid in depleting the ozone layer, non-renewable(can quickly get diminished), fluctuation prices etc. As a result, these traditional forms are struggling to keep up with the rapid growth of the population Sharafi (2014). Therefore, building a much more sustainable and cost-effective energy supply system is highly required ( $\mathrm{Ji}, 2011)$. Among the different forms of energy, Renewable Energy Sources (RES) such as solar, wind, biomass, etc. seem to be the most viable alternative (R. Shah, 2017). The use of the RES could reduce the effects associated with the use of conventional energy sources to the minimum barest (Magazine, 2020) and as a result, many countries have embraced the integration of renewable sources of energy into the conventional power system. According to Abbassi (Abbassi et al., 2018), the total fraction of RES in global energy is on the high increase. Among the RES, especially in areas where the sun's intensity is immense, the most promising RES is Solar energy, especially Solar Photovoltaic (PV) technology (Gao et al., 2018). The PV module overall output highly is governed by its rated power output, the intensity of the sun's radiation (Irradiance), cell temperature, efficiency, amount of shading experienced by the system and the positioning (tilt and azimuth angles) (Ghaedi et al., 2014).

PV panel's efficiency conversion rate and its performance mostly depend on its physical parameters (Abbassi et al., 2018). Some of these parameters are given under the standard test condition and others have to be determined (Abbassi et al., 2018). Most researchers, for instance, assume that the diode ideality factors are given as $n_{1}=1, n_{2}=2$. 
Although this assumption is widely used it is not always true especially for panels with larger capacities (Ishaque et al., 2011a).

In most recent times, many mathematical models representing the dynamics of the PV Cell have been formulated and the single diode model is the commonest and the simplest one (Ishaque et al., 2011b). In this model, only three basic parameters, that is, Short-circuit Current $\left(I_{S}\right)$, Open-Circuit Voltage $\left(V_{o}\right)$ and the diode ideality factor. These models cannot contain temperature variations (Islam, 2014). The inclusion of the additional diode solves this problem but it increases the parameters to seven, that is, the PV photo-current $\left(I_{P H}\right)$, the Diode's current $\left(I_{s 1}, I_{s 2}\right)$, Series $\left(R_{s}\right)$, Shunt $\left(R_{p}\right)$ resistance, Ideal diode factors $\left(n_{1}, n_{2}\right)$ (Gao et al., 2018). Appropriate estimation of these parameters at various values of irradiance and temperature conditions help maximize the energy production of PV module (Ishaque et al., 2011a). Using the two-diode model though increases the complexity of the problem it gives much more accurate and realistic results (Islam, 2014). Therefore, a precise estimation of the parameters is very important as it would ensure an accurate design of the PV system. Nevertheless, this extraction remains a complex stochastic nonlinear optimization problem due to the nonlinear nature of the double-diode equations and the variability of the meteorological data (Abbassi et al., 2018). Abbassi Abbassi et al. (2018) stated that all the parameters to be estimated are highly sensitive to the intermittency in the irradiance and temperature which also affect the PV power output. Considering the uncertainty would make the results much more realistic Abbassi et al. (2018). The main challenge now is to estimate the values of all the model parameters accurately while maintaining a reasonable computational effort.

According to a thorough review by Abbassi et al. (2018) and others, a series of extensive studies that have been carried out to deal with such problem using analytical, optimization, artificial intelligence, and other statistical methods. However, there are still more researches that need to be carried out to achieve a minimum error and a high accuracy (Ishaque et al., 2011b,a; Lim et al., 2015; Liu and Zhang, 2018). For instance, in the study conducted by Lim et al. (2015), it was proved that it is impossible to get an accurate solution to the nonlinear problem by an analytical approach. Artificial Neural Network (ANN) approaches turn to be computationally expensive (Islam, 2014). Abbassi et al. (2018) concluded that though a lot of optimization methods have been applied over the years, Comparisons of different hybrid optimization algorithms are required for obtaining better results. 
Academic Journal of Research and Scientific Publishing | Vol 3 | Issue 28

Publication Date: 5-8-2021 ISSN: 2706-6495

None of these researches has considered the stochasticity of the meteorological data in the extracting of these parameters (Abbassi et al., 2018). Therefore in this study, the objective is to apply a stochastic optimization to deal with the uncertainty and compare various hybrid optimization methods to identify the most promising algorithm for extracting the optimal values of the $n_{1}, n_{2}, R_{s}$ and $R_{p}$ that would yield maximum power.

\section{Methodology}

\subsection{How the PV Cell Works}

Every PV cell is made up of a semiconductor diode with its $\mathrm{p}-\mathrm{n}$ junction being visible to sunlight. PV cells can be made from different types of semiconductors. In this study, the focus is on silicon cells due to their availability on the market (Ghaedi et al., 2014). Silicon PV cells are made up of a thin Si film joined to electric terminals with one side doped to form the $\mathrm{p}-\mathrm{n}$ junction. The general operation of a PV cell is based on the photoelectric effect principle. The photoelectric effect refers to the ejection of a conduction band due to the constant absorption of sunlight (Hanifi et al., 2020). Thus, when the sunlight hits the cell's surface, a proportion of the solar radiation is absorbed in the semiconductor material. When the energy absorbed exceeds the bandgap energy, electrons from the valence band moves to the conduction band. Consequently, electron-hole pairs are formed illuminated region of the semiconductor and these electrons conduction bands are now free to move. The electric field in the PV cell is used to regulate the moving electrons in a specific direction. The movement of the electrons form the PV current (Islam, 2014). Figure 1 depicts the physical structure of a PV cell. 


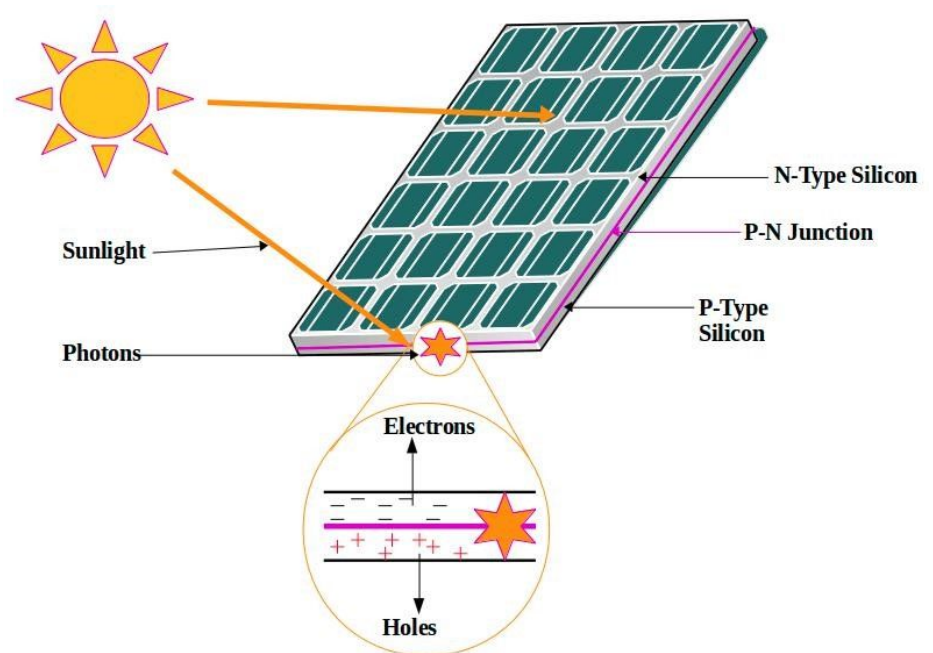

Figure 1: PV panel.

\subsection{The Current-Voltage (I-V) Curve}

These curves depict the geometry of the operation of a solar panel summarizing the relationship between the PV current and voltage under variable conditions of Solar irradiance and temperature. The curve gives basic details on how to set up and regulate a PV system to obtain the maximum power output (Islam, 2014). To be able to perform a simulation on the PV cell, analyze its dynamics and obtain the objective function to be optimized, it is vital to obtain the double-diode PV cell model.

\subsection{The Double Diode-Mathematical Model of PV Cell}

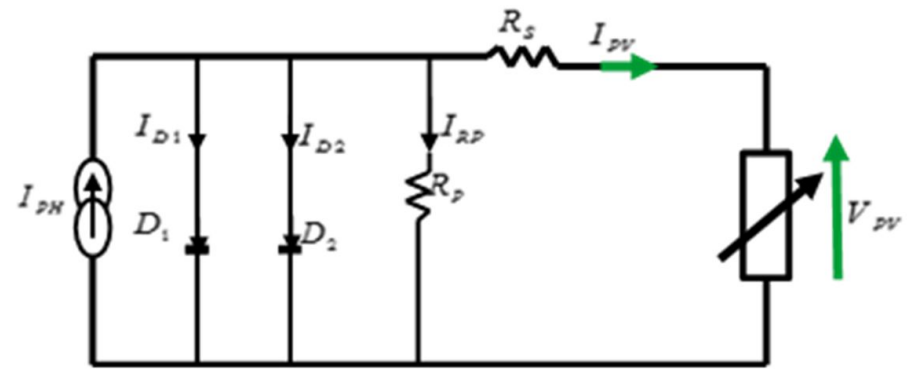

Figure 2: PV cell equivalent circuit 
Academic Journal of Research and Scientific Publishing | Vol 3 | Issue 28

Publication Date: 5-8-2021 ISSN: 2706-6495

By applying Kirchhoff's current law to Figure 2, we have (Abbassi et al., 2018):

$$
I_{P V}=I_{P H}-I_{D 1}-I_{D 2}-I_{R P}
$$

where the shunt current $I_{R P}$ is given by Islam (2014):

$$
I_{R P}=\frac{V_{P V}+I_{P V} R_{S}}{R_{P}}
$$

The PV photo-current $\left(I_{P H}\right)$, which depends on the solar insolation and cell's temperature is given by Nguyen (2015):

$$
I_{P H}=\left[I_{S C}+T_{i}\left(T_{c}-T_{r}\right)\right] \frac{I_{T}}{1000}
$$

And the cell temperature $\left(T_{c}\right)$, is given as Islam (2014):

$$
T_{c}=T_{a}+\left(\frac{T_{N}-20}{80}\right) I_{T}
$$

Where

$I_{s c}$ represents the current of the short circuit, $I_{T}$ denotes the global solar irradiance, $T_{i}$ is the temperature coefficient, $T_{r}$ denotes temperature reference, $T_{a}$ denotes the ambient temperature, $R_{s}$ denotes the Series resistance, $R_{p}$ denotes the Shunt resistance, $T_{N}$ denotes the Nominal Operating Temperature of the cell.

The mathematical model for estimating the total solar irradiance on the sloped PV panel is the Isotropic model developed by Lui and Jordan (2002):

$$
I_{T}=\left(\frac{\cos \left(\theta_{i}\right)}{\cos \left(\theta_{z}\right)}\right) E_{H b}+\left(\frac{1+\cos (\beta)}{2}\right) E_{H d}+\left(\frac{1-\cos (\beta)}{2}\right) \rho_{g} E_{H r}
$$

Where

$$
\begin{aligned}
& I_{P H}=\left[I_{s c}+T_{i}\left(T_{c}-T_{r}\right)\right] \frac{I_{T}}{1000} \\
& \cos \theta_{i}=\cos (\phi-\beta) \cos \delta \cos \omega+\sin (\phi-\beta) \sin \delta
\end{aligned}
$$

$E_{\mathrm{Hb}}$ is the beam irradiance, $E_{\mathrm{Hd}}$ is diffused irradiance, $E_{\mathrm{Hr}}$ is the reflected irradiance, $\rho_{g}$ is the albedo $\in[0.2,0.7], \omega$ is the hour angle, $\delta$ is the declination angle of the sun, $\varphi$ is the latitude, $\theta_{i}$ is the angle of incidence, and $\theta_{z}$ is the zenith angle of the panel.

Also, the Diode 1 Current is given Elbaset et al. (2015):

$$
I_{D 1}=I_{s 1}\left[\exp \left(\frac{V_{P V}+I_{P V} R_{S}}{n_{1} V_{t 1}}\right)-1\right]
$$


and Diode 2 Current is:

$$
I_{D 2}=I_{S 2}\left[\exp \left(\frac{V_{P V}+I_{P V} R_{S}}{n_{1} V_{t 1}}\right)-1\right]
$$

Diodes thermal voltages can be estimated by:

$$
V_{t 1}=V_{t 2}=V_{t}=\frac{N_{s} k T_{c}}{q}
$$

where $N_{s}$ and $N_{p}$ are the number of panels joined in series and parallel to step up the PV voltage and current respectively.

Several researchers assume that the diode factors $n_{1}=1$ and $n_{2}=2$ for $n_{1}, n_{2} \in[1,2]$ and though this assumption is widely used it is not always true especially for panels with larger capacities (Ishaque et al., 2011a). Thus, in this study, the parameters $R_{p}, R_{s}, n_{1}, n_{2}$ are the decision variables that are to be determined using an optimization approach. The Panel's saturation currents, $I_{s 1}$ and $I_{s 2}$, computed by (Ishaque et al., 2011b):

$$
I_{s 1}=I_{s 2}=\frac{I_{S C}+T_{i}\left(T_{C}-T_{r}\right)}{\exp \left[\frac{V_{o c}+T_{v}\left(T_{C}-T_{r}\right)}{V_{t}}\right]-1}
$$

where $\mathrm{q}$ is the electron charge $=1.6 \times 10^{-19} C, V_{o c}$ is the open circuit voltage $(\mathrm{V}), n_{1,2}$ are the ideality factors of the diodes, $T v$ is the Temperature coefficient of open circuit voltage, $\mathrm{k}$ is the Boltzmann's constant $\left(1.3805 \times 10^{-23} \mathrm{~J} / \mathrm{K}\right)$. From Equations (1), (2), (6) and (7), Equation (1) becomes:

$$
\begin{gathered}
I_{P V}(t)=\left[I_{s c}+T_{i}\left(T_{c}-T_{r}\right)\right] \frac{I_{T}}{1000}\left\{\frac{I_{S C}+T_{i}\left(T_{C}-T_{r}\right)}{\exp \left[\frac{V_{o c}+T_{V}\left(T_{C}-T_{r}\right)}{V_{t}}\right]-1}\right\} \\
\left\{\left[\exp \left(\frac{V_{P V}+I_{P V} R_{S}}{n_{1} V_{t 1}}\right)-1\right]-\left[\exp \left(\frac{V_{P V}+I_{P V} R_{S}}{n_{2} V_{t 2}}\right)-1\right]\right\}-\frac{V_{P V}+I_{P V} R_{S}}{R_{P}}
\end{gathered}
$$

And the output voltage can deduced from Equation (6) as(Islam, 2014):

$$
\begin{gathered}
V_{P V}(t)=\left(\frac{V_{t 1} V_{t 2}}{V_{t 1}-V_{t 2}}\right) \\
\ln \left[\exp \left(\frac{-\left(V_{P V}+I_{P V} R_{S}\left(N_{s} / N_{p}\right)\right)}{V_{t 2}}\right)\left\{\frac{I_{P H}+2 I_{S 1}-I_{P V}}{I_{S 1}}-\left(V_{P V}+I_{P V} R_{S} \frac{\left(N_{S} / N_{p}\right)}{I_{S 1} R_{p}\left(N_{S} / N_{p}\right)}\right)\right\}-1\right]-I_{P V} R_{S}\left(N_{S} / N_{p}\right)
\end{gathered}
$$


Academic Journal of Research and Scientific Publishing | Vol 3 | Issue 28

Publication Date: 5-8-2021 ISSN: 2706-6495

These equations are used to describe the I-V characteristics curve and the objective function to be optimized is given as (Islam, 2014):

$$
P_{t}=I_{P V}(t) \times V_{P V}(t) \times E_{P V}
$$

where $E_{p v}$ is the panel's efficiency.

In addressing the uncertainty issue in optimization, many stochastic optimization methods exist. Among these, the Monte-Carlo method (Sample Average Approximation) and Chance Constraint(CC) approaches are the commonest and have many promising real life applications. However, the main issues with the $\mathrm{CC}$ are that it assumes that the randomness follows a bi-variant Gaussian distribution and restricts the search space which consequently makes the problem solution more conservative and basically low efficient (Kamjoo, 2015). Therefore in this study, the Sample Average Approximation (SAA) is employed to handle the uncertainty due to its robustness and effective handling of nonlinear problems. The summary of this approach is discussed in the next section.

\subsection{Sample Average Approximation}

The objective function given by Equation (13) can be rewritten as, for $\mu=1,2, \ldots$, and $\alpha$ is the uncertainty parameter (meteorological data). Direct optimization of $F_{\mu}\left(x, \alpha_{\mu}\right)$ is not possible due to high number of scenarios and thus its expected value of the form, $E\left(F_{\mu}\left(x, \alpha_{\mu}\right)\right)$ is targeted (Birge, 2011).

Assuming a sample of realizations, $\alpha^{1}, \ldots, \alpha^{M}$ of the random vector $M$, after the Monte Carlo simulations, the function $F_{\mu}\left(x, \alpha_{\mu}\right)$ is transformed as Birge (2011):

$$
F_{\mu}=\frac{1}{M} \sum_{\mu=1}^{M} f_{\mu}\left(x, \alpha_{\mu}\right)
$$

Applying the Law of Large Numbers gives:

$$
\frac{1}{M} \sum_{\mu=1}^{M} f_{\mu}\left(x, \alpha_{\mu}\right) \approx E F_{\mu}\left(x, \alpha_{\mu}\right)
$$


Academic Journal of Research and Scientific Publishing | Vol 3 | Issue 28

Publication Date: 5-8-2021 ISSN: 2706-6495

A deterministic equivalent of the stochastic problem is given as:

$$
\max \left(\frac{1}{M} \sum_{\mu=1}^{M} f_{\mu}\left(x, \alpha_{\mu}\right)\right) x \in K
$$

Therefore, the optimization problem can be written as:

$$
\begin{aligned}
x=\left[n_{1}, n_{2}, R_{s}, R_{p}\right] & \text { minimize } \\
& x_{\min } \leqslant x \leqslant x_{\max }
\end{aligned}
$$

Where $x_{\min }, x_{\max }$ are the highest and least values of the decision variables.

In the next section, various optimization algorithms are employed to extract the PV cell physical parameters.

\subsection{Particle Swarm Optimization (PSO)}

PSO is a population-based algorithm motivated by the mass movement and social behaviour of particles such as the swarm of bees, school of fish, etc. (Parizad et al., 2010). It is a random optimization algorithm based on swarm aptitude. Figure 3 summarizes the general movements of the particle in searching for the solution within a solution space.

The following describes the principle behind the search process (Del Valle et al., 2008):

- Employs a couple of particles that form the swarm to explore the search space and find the most optimal solution.

- Every particle modifies the movement by its own experience and that of others and consequently navigate to a better space to get the best solution.

- The following records are kept by each of the particles: particle own the best position $\left(P_{\text {best }}\right)$, best of all the particle's position $\left(g_{\text {best }}\right)$.

- The position of each particle is modified by: its new position, new velocity, the distance between its new position and $(\mathbf{p}(t))$, and distance between its new position and $(\mathbf{g}(t))$.

Geometrically, this is represented in Figure 3: 
Academic Journal of Research and Scientific Publishing | Vol 3 | Issue 28

Publication Date: 5-8-2021 ISSN: 2706-6495

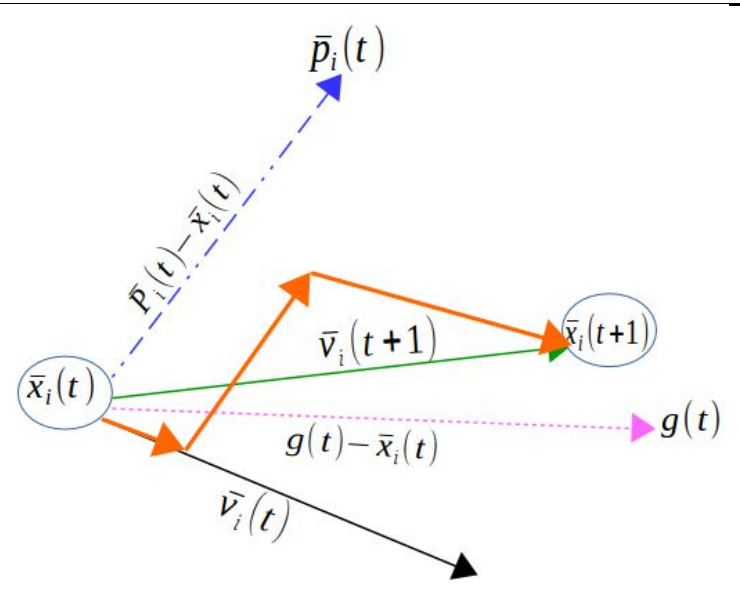

Figure 3: The Geometrical Representation of PSO

Analytically, the current velocity and position are given by:

$$
\begin{aligned}
& \overrightarrow{v_{l}}(t+1)=w \overrightarrow{v_{l}}(t)+r_{1} c_{1}\left[\overrightarrow{p_{l}}(t)-\overrightarrow{x_{l}}(t)\right]+r_{2} c_{2}\left[\overrightarrow{g_{J}}(t)-\overrightarrow{x_{l}}(t)\right] \\
& \overrightarrow{x_{l}}(t+1)=\overrightarrow{x_{l}}(t)+\overrightarrow{v_{l}}(t+1)
\end{aligned}
$$

Where: $c_{1}, c_{2}$ are the coefficients of acceleration which attracts a towards $\vec{p}(t)$ and $\vec{g}(t)$ respectively. The $r_{1}, r_{2} \sim U(0,1)$ and $\mathrm{w}$ denotes the weight of inertia which is basically used to ensure a balance between the $\vec{p}(t)$ and $\vec{g}(t)$ (Tian, 2018). Higher $\mathbf{w}$ values enhance the search of $\vec{g}(t)$ and lower values, facilitates the search of the $\vec{p}(t)$ around the new search space. The general mechanism is summarized in Figure 4 


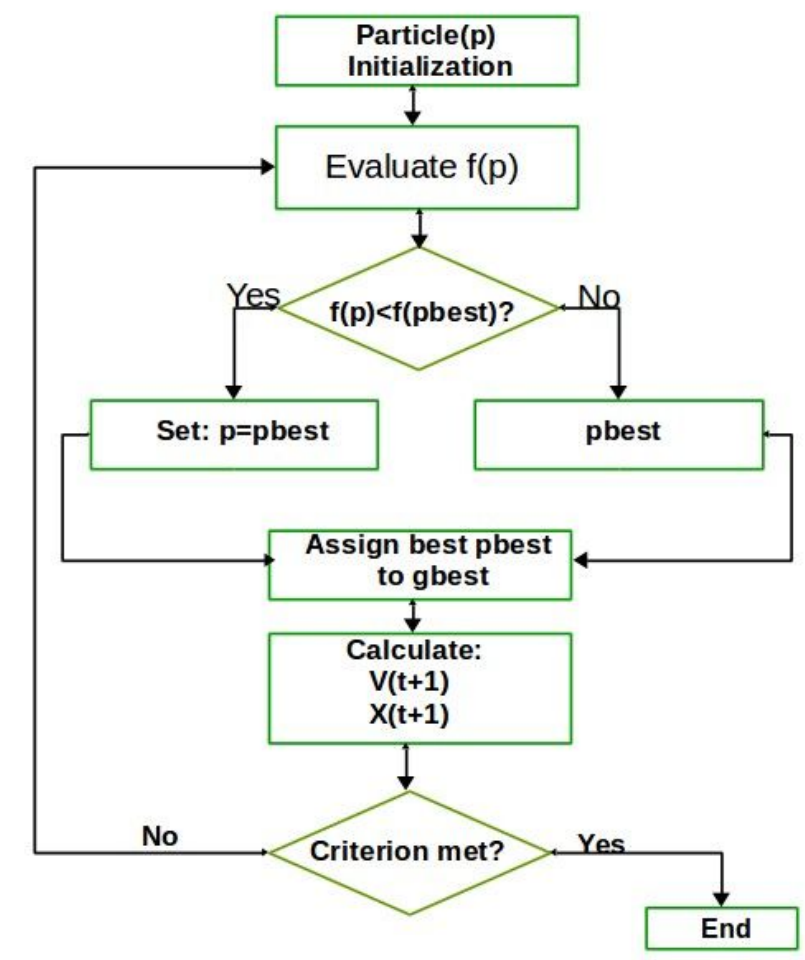

Figure 4: The flowchart of PSO

\subsection{The Mechanism of Grey Wolf Optimizer (GWO)}

The GWO is a recent meta-heuristic algorithm that was developed in 2014 by (Mirjalili et al., 2014). This algorithm is based on the principle of grey wolves hunting, that is, it deals with the modelling of grey hunting. The general social mechanisms of GWO is classified into four categories (Mirjalili et al., 2014):

- The alphas (dominant wolves) are the main leaders or in the search process. They are responsible for making major decisions like deciding the spots for resting, the sleeping or waking time, and the entire hunting process. In general, the vision or intentions of the dominant wolves are commanded to the group.

- The second most powerful class of wolves are the beta grey wolves. This class consults the alphas and give support to lead and make a decision that would enhance the search process. 
Academic Journal of Research and Scientific Publishing | Vol 3 | Issue 28

Publication Date: 5-8-2021 ISSN: 2706-6495

- Apart from the two most powerful classes(alpha and beta), the next most powerful level is the omega. They mostly serve as scapegoats. They obey the alpha and beta wolves. They have to eat after all. When there are no omega wolves, there will be civil war and troubles.

- The Delta wolves are the last level. They used to be either alpha or beta wolves. They must surrender to the alpha and beta but overpowers the omega class. They mostly render support to others when the need arises (Mirjalili et al., 2014).

During hunting, the Gray wolves envelop the hunt and gradually approaches the hunt till it stops and eventually attacks it. At the end of the search process, the alphas are considered as the most optimal solution follows by the betas, $\delta$ and then omega or others (Miao et al., 2020).

Now the mathematics behind this algorithm is given as follows: If vector $\overrightarrow{x_{p(t)}}$ denotes the prey's position, then position of the grey wolves is given in Equations (19) and (20) as (Miao et al., 2020):

$$
\begin{aligned}
& \overrightarrow{x_{l}}(t+1)=\overrightarrow{x_{p(t)}}-\vec{U} \cdot \vec{D} \\
& \vec{D}=\left|\overrightarrow{c x_{p(t)}}-\overrightarrow{x_{(t)}}\right|
\end{aligned}
$$

where $t$ is the number of iterations, the factor vectors $(\vec{U}, \vec{C})$ can be found using the Equations (22) and (23):

$$
\begin{gathered}
\vec{U}=2 \vec{r} \overrightarrow{r_{1}}-\vec{a} \\
\vec{C}=2 \overrightarrow{r_{2}}
\end{gathered}
$$

or the attributes of a decreasing from 2 to 0 over the iteration period. $\overrightarrow{r_{1}}$ and $\overrightarrow{r_{2}}$ represent random vectors in $[0,1]$. The best solutions are the alpha, beta and deltas in that order. Others update their position like best solutions. These are presented in Equations (24) - (30):

$$
\begin{aligned}
& \overrightarrow{D_{\alpha}}=\left|\overrightarrow{c_{1} \cdot x_{\alpha}}-\vec{x}\right| \\
& \overrightarrow{D_{\beta}}=\left|\overrightarrow{c_{2} \cdot x_{\beta}}-\vec{x}\right| \\
& \overrightarrow{D_{\delta}}=\left|\overrightarrow{c_{3} \cdot x_{\delta}}-\vec{x}\right|
\end{aligned}
$$




$$
\begin{aligned}
& \overrightarrow{x_{1}}=\left|\overrightarrow{x_{\alpha}}-\overrightarrow{A_{1}} \overrightarrow{D_{\alpha}}\right| \\
& \overrightarrow{x_{2}}=\left|\overrightarrow{x_{\beta}}-\overrightarrow{A_{2 .}} \overrightarrow{D_{\beta}}\right| \\
& \overrightarrow{x_{3}}=\left|\overrightarrow{x_{\delta}}-\overrightarrow{A_{3}} \overrightarrow{D_{\delta}}\right| \\
& \overrightarrow{x_{(t+1)}}=\frac{\overrightarrow{x_{1}}+\overrightarrow{x_{2}}+\overrightarrow{x_{3}}}{3}
\end{aligned}
$$

The procedures of the GWO are outlined in Figure 5.

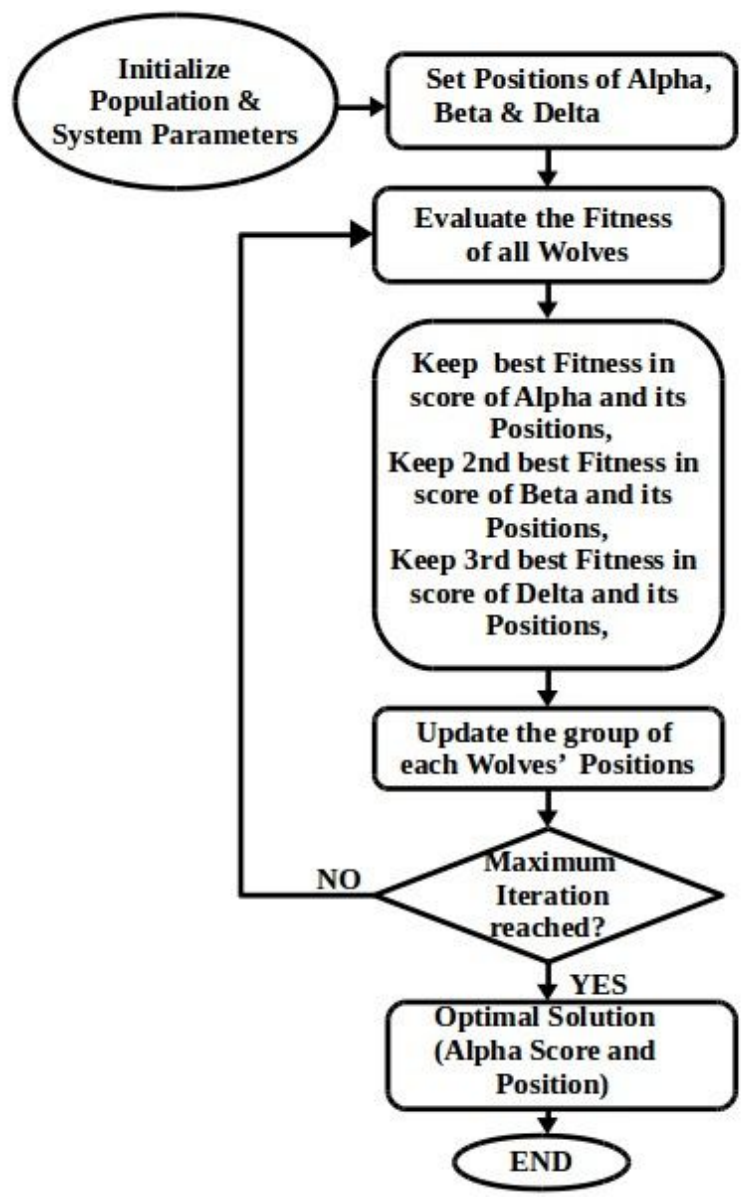

Figure 5: The mechanism of GWO

\subsection{Hybrid Particle Swarm Grey Wolf Optimization Algorithm (Hybrid PSO-GWO)}

Generally, most optimization algorithms get stuck in local optimum (suffers premature convergence) or have a slow rate of convergence when dealing with highly complex problems whose nature is nonlinear, non-convex, non-smooth, etc. 
Some algorithms better explore the search space and have a slow convergence rate while some algorithms less diversely explore the search space and did not find the optimal solution. Maintaining the balance between exploration and exploitation calls for the integration of algorithms to form a hybrid algorithm. In this study, a Hybrid PSO-GWO Algorithm developed by (Senel et al., 2019) has been applied to extract the physical parameters from the double diode nonlinear PV cell equation presented in Equation (11). The PSO mostly gets stuck in local optimum and thus, the GWO algorithm is employed to avoid getting stuck in a local optimum (Senel et al., 2019). The Particle Swarm algorithm assigns particles to random positions with a small chance of avoiding getting stuck in a local optimum. These random placements might force the search to move away from the global optimum.

The GWO has a great exploration capacity that could be used to direct the particles to positions that are partially improved by the GWO instead of directing them to random positions which would consequently reduce the risks of getting stuck in a local optimum. The general search approach has been outlined in Figure 6.

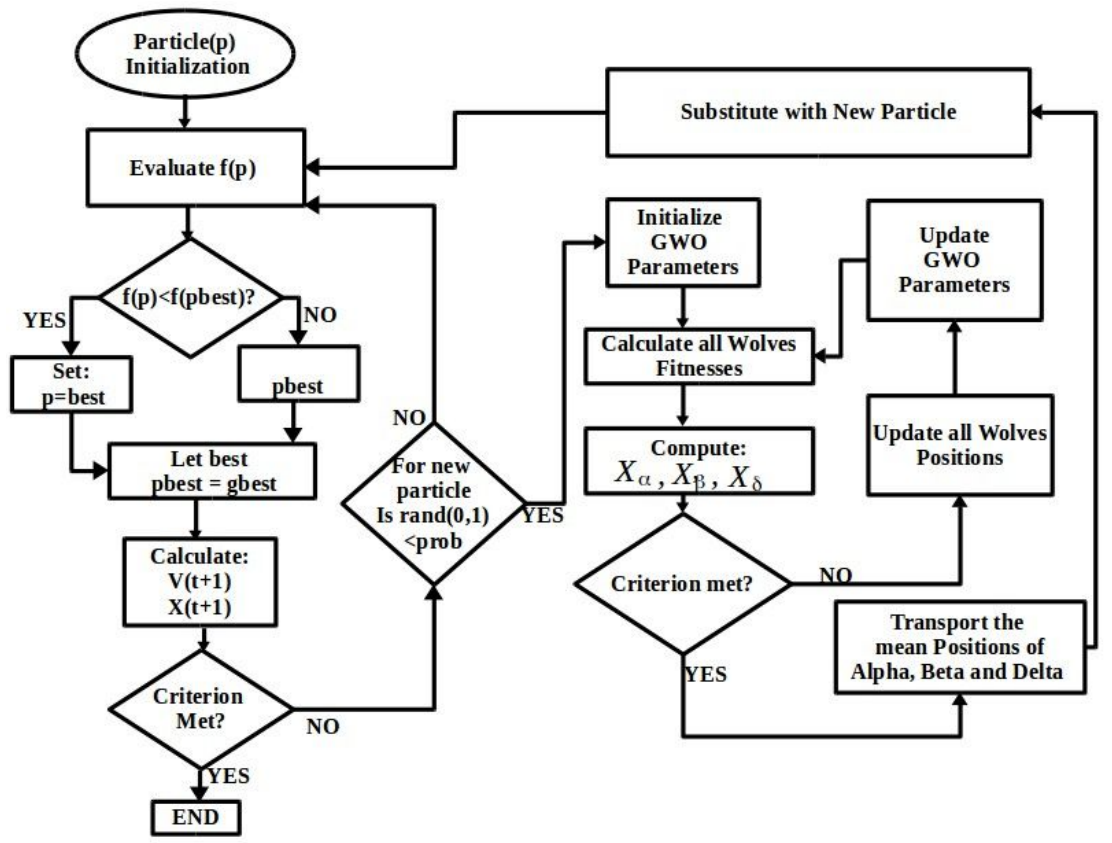

Figure 6: The flowchart of the Hybrid PSO-GWO algorithm. 
Academic Journal of Research and Scientific Publishing | Vol 3 | Issue 28

Publication Date: 5-8-2021 ISSN: 2706-6495

\section{Simulation, Results and Discussion}

At this section, the two-diode mathematical models of the PV cell have been implemented using the parameters in Table 2. Also, the amount of solar irradiance defined by Equations (5) has been estimated using the measured data from the KNUST energy centre and it is depicted by Figure 7. It was assumed that the average temperature at KNSUT is given as [27.03,29.46,29.97,29.55,28.58,27.13,26.09,25.69,26.22,27.09,27.57,26.89] across the months.

Monte-Carlo simulation was carried out to handle the random objective function, that is, estimating the mean values of the PV power. The impact of intermittency in temperature and solar irradiance is examined. The optimization algorithms are applied to select the optimal parameters. Finally, the results obtained are analyzed and discussed. Both the simulations and the optimization approaches are performed in MATLAB environment on $2.87 \mathrm{GHz}$ and Core i7 processor with 4 GB of RAM.

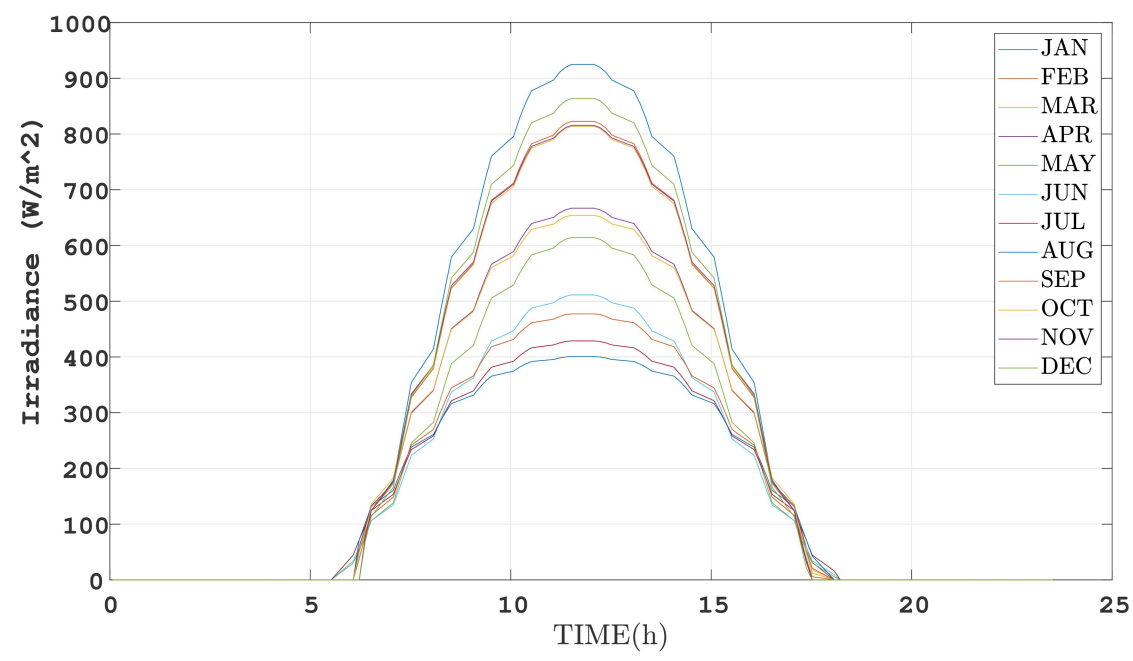

Figure 7: Estimated Irradiance on a PV Panel at KNUST 
Academic Journal of Research and Scientific Publishing | Vol 3 | Issue 28

Publication Date: 5-8-2021 ISSN: 2706-6495

Table 1: Specifications of the PV Silicon Cell system in Ghana.

\begin{tabular}{|c|c|c|c|}
\hline Parameter & Estimated Value & Source & Unit \\
\hline $\operatorname{Efficiency}(\eta)$ & 19.60 & Djamila (2018) & $\%$ \\
\hline \multirow[t]{2}{*}{ Open circuit voltage $\left(V_{o c}\right)$} & 64.80 & Sunpower & $\mathrm{V}$ \\
\hline & & $(2020)$ & \\
\hline \multirow[t]{2}{*}{ Short circuit current $\left(I_{s c}\right)$} & 6.24 & Sunpower & A \\
\hline & & $(2020)$ & \\
\hline Longitude & $1.5654^{\circ} \mathrm{W}$ & & \\
\hline Latitude $(\varphi)$ & $6.6732^{\circ} \mathrm{N}$ & & \\
\hline Reflectance $(\rho)$ & 0.2 & & \\
\hline Tilt angle $(\beta)$ & $30^{\circ}$ & & \\
\hline Azimuth angle $(\gamma)$ & 0 。 & & \\
\hline \multirow[t]{2}{*}{ Temperature coefficients ()} & -0.38 & Sunpower & $\% / \mathrm{K}$ \\
\hline & & $(2020)$ & \\
\hline \multirow[t]{2}{*}{ Voltage $(V)$} & -176.60 & Sunpower & $\mathrm{mV} / \mathrm{K}$ \\
\hline & & $(2020)$ & \\
\hline \multirow[t]{2}{*}{ Current/temperature coefficient $(I)$} & 3.50 & Sunpower & $\mathrm{mV} / \mathrm{K}$ \\
\hline & & $(2020)$ & \\
\hline Voltage/temperature coefficient & $-80 \times 10^{-3}$ & & $(\mathrm{~V} / \mathrm{K})$ \\
\hline Number of Connected series cells $\left(N_{s}\right)$ & 36 & & \\
\hline Number of Connected parallel cells & 25 & & \\
\hline \multirow[t]{2}{*}{ NOCT } & $45 \pm 2$ & Sunpower & Celcius \\
\hline & & (2020) & \\
\hline
\end{tabular}

\subsection{The Monte Carlo Simulations}

Prior to the implementation of Monte-Carlo simulations to estimate the expected mean power value $\left(E\left(F_{\mu}\left(x, \alpha_{\mu}\right)\right)\right)$, the uncertainties in the problem was modeled by fitting the historical 
Academic Journal of Research and Scientific Publishing | Vol 3 | Issue 28

Publication Date: 5-8-2021 ISSN: 2706-6495

meteorological data to a probability distribution to serve as random data generator in Monte Carlo simulation for a realistic design output Kamjoo (2015). Selection of the most appropriate distribution completely depends on the location so to obtain the most suitable probability distribution, two standard probability functions, Weibull and Beta, were compared (Kamjoo, 2015). The results indicated that the Beta with $R M S E=0.3306$ outperformed the Weibull with $R M S E=0.4167$ after their outputs were compared with the output of the actual data and this is demonstrated by the Figure 8 .

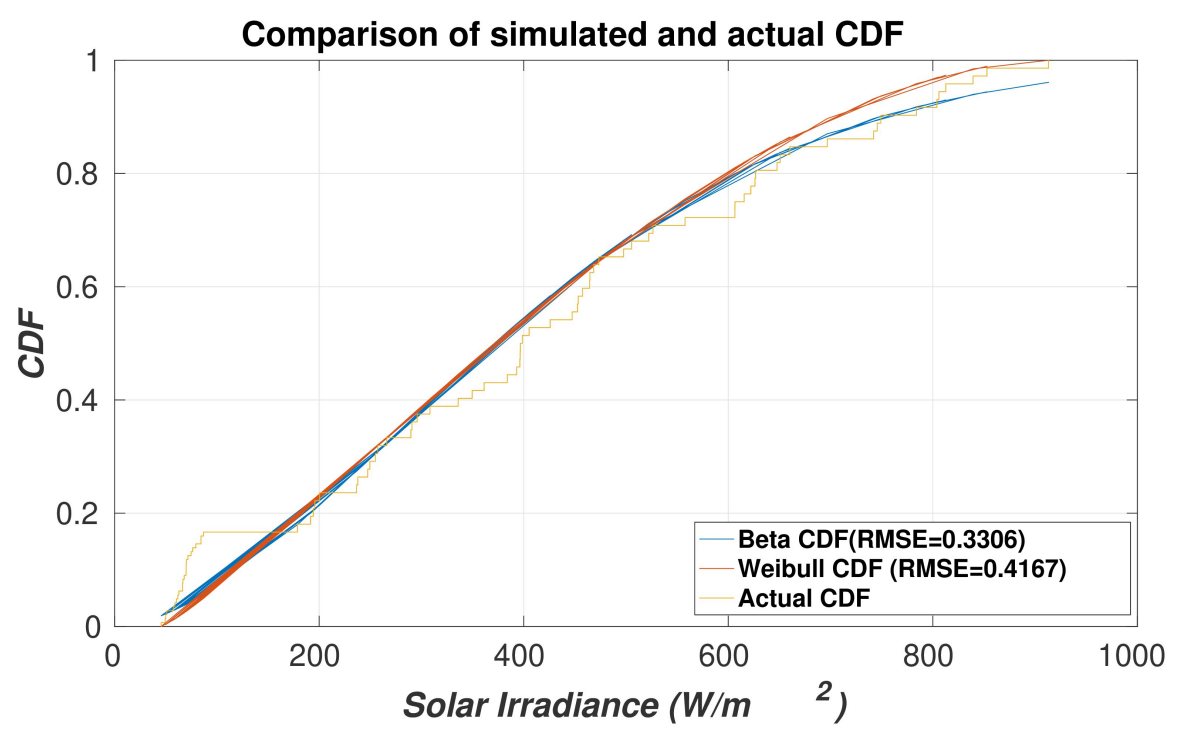

Figure 8: RMSE of Weibull (0.4167) and Beta (0.3306).

After running the Monte Carlo simulations for a sample of (10000), the results in Figure 9) show that the converged mean value of the Power from the Monte Carlo simulations gets closer to the average of the actual data as the sample size gets larger which establishes the law of large numbers stated previously. 


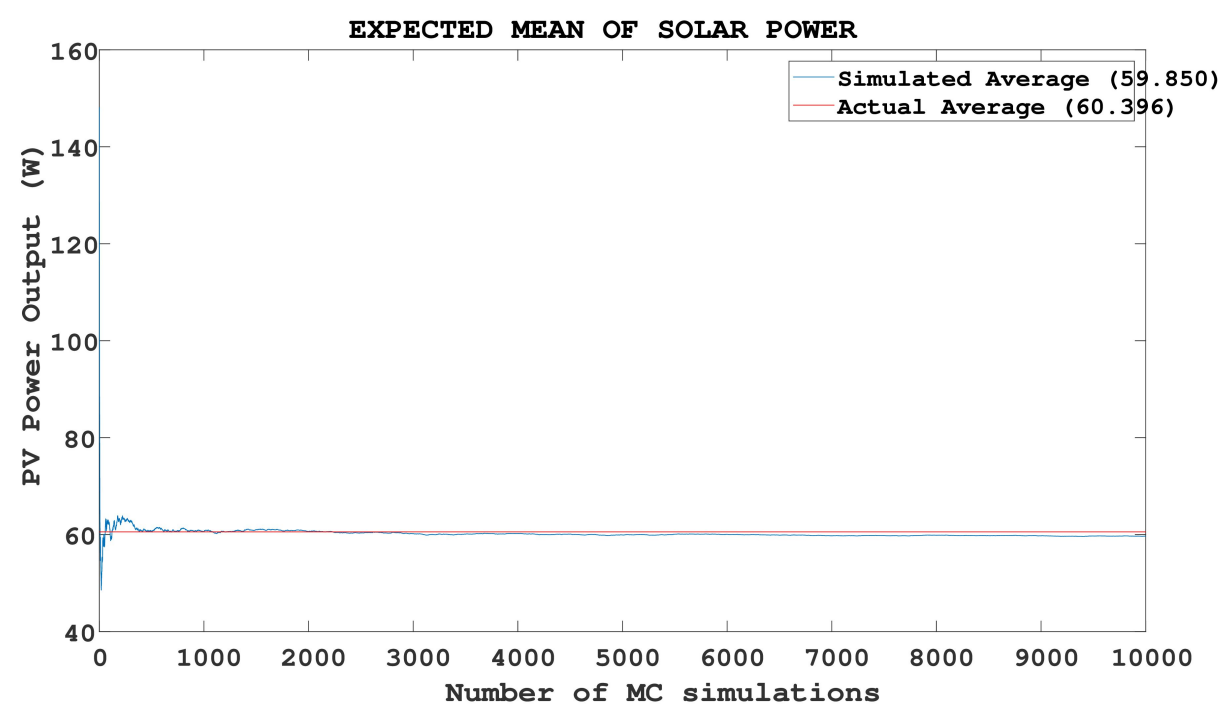

Figure 9: Expected mean of PV Power from Monte Carlo simulations

\subsection{The Optimization Module}

In this study, different algorithms such as the GA, PSO, GWO, Hybrid GA-PSO (HGAPSO), Hybrid PSO-GWO (HPSO-GWO), Hybrid GWO-Sine Cosine (HGWOSCA) have been applied to the problem. The general assessment is given in the table (2). The initial population (Num. Pop.) and the maximum number of iterations (Max. IT) for all the algorithms were chosen as 100 and 500 respectively. The limits for the decision variables are given as: $0.5 \leq n_{1}, n_{2} \leq 2 ; 0 \leq$ $R_{s} \leq 2 ; 200 \leq R_{s h} \leq 500$. From the results, it can be observed that the HPSO-GWO produced the most optimal values $\left(R_{S}=1.4623, R_{s h}=215.0000, n_{1}=0.9500, n_{2}=1.6500\right)$ of the parameters since it produced a higher power value $(59.850 \mathrm{~W})$. The CPU execution time of the HPSOGWO and others were quite higher and this is due to the use of the Monte-Carlo simulation in dealing with the randomness effect in the problem.

Table 2: The GA, PSO, GWO, HGAPSO, HPSOGWO and GWOSC results.

\begin{tabular}{lllllll}
\hline Variables & GA & PSO & GWO & HGAPSO & $\begin{array}{l}\text { HPSO- } \\
\text { GWO }\end{array}$ & HGWOSC \\
\hline$R_{s}$ & 1.4673 & 1.0887 & 1.7000 & 1.3081 & 1.4623 & 1.2778 \\
\hline
\end{tabular}


Academic Journal of Research and Scientific Publishing | Vol 3 | Issue 28

Publication Date: 5-8-2021 ISSN: 2706-6495

\begin{tabular}{|c|c|c|c|c|c|c|}
\hline$R_{s h}$ & 200.000 & 203.60 & 205.00 & 202.00 & 215.00 & 201.00 \\
\hline$n_{1}$ & 1.0500 & 1.0000 & 1.2000 & 1.0000 & 0.9500 & 1.0900 \\
\hline$n_{2}$ & 1.9400 & 1.8800 & 1.9100 & 1.9000 & 1.6000 & 1.6500 \\
\hline CPU(s) & 37.5430 & 28.0190 & 28.4980 & 45.1870 & 56.6670 & 58.4620 \\
\hline Max. IT & 500 & 500 & 500 & 500 & 500 & 500 \\
\hline PMax & 52.180 & 53.883 & 52.02 & 54.878 & 59.850 & 54.862 \\
\hline Num. Pop. & 100 & 100 & 100 & 100 & 100 & 100 \\
\hline
\end{tabular}

In order to simulate the double-diode model to produce the I-V curve, the mean values of the solar irradiance and the temperature in Figure 7, the data in Table 2 and the optimal values obtained from the optimization module were used. The results are as follows. The Figure 10 demonstrates the I-V curve of an actual solar silicon cell working at standard conditions. Every point on the curve

represents the PV power output at different points. When the PV is open-circuited which implies that no load is connected, the system current is approximately null whilst its voltage is at peak. On the other hand, if the cell is in short-circuit, the PV current passing out of it would be at its peak and the voltage will approximately be zero. This is depicted by Figure 11.

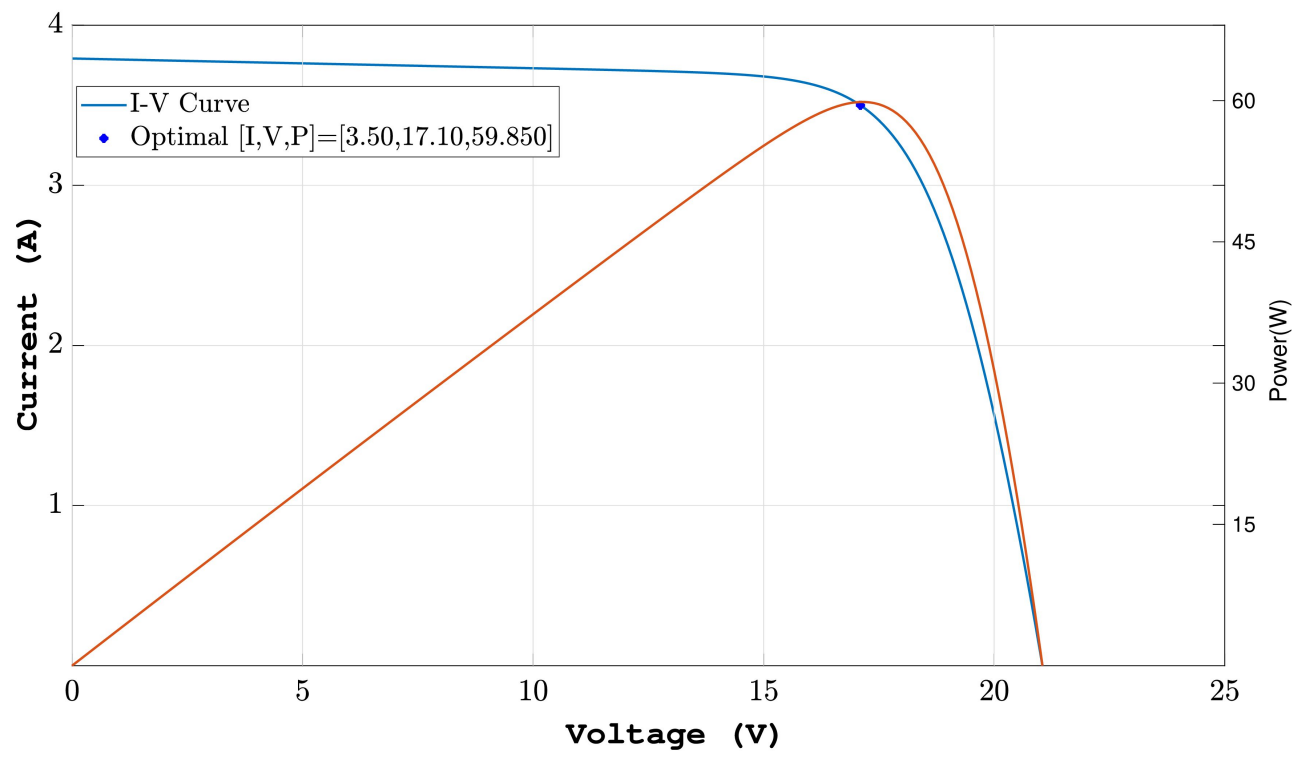

Figure 10: The Current-Voltage characteristics curve. 
Academic Journal of Research and Scientific Publishing | Vol 3 | Issue 28

Publication Date: 5-8-2021 ISSN: 2706-6495

Conversely, the peak voltage obtained from the PV cell is at its open-circuit but the peak current is at the closed circuit. It can be seen that the maximum power is not obtained at any of these conditions. Nevertheless, there exist one point on the I-V characteristics curve at where the maximum PV power could be obtained, that is, $\left(I_{m p}, V_{m p}\right)$. Obtaining this point on the curve solely relies on controlling of the variations in the meteorological information and optimal values of the cell parameters. Therefore, the optimization algorithms were used to get these optimal values and the Monte-Carlo was used to handle the randomness. The maximum power delivered by a PV cell at current and voltage is given as $P_{t}=I_{P V} \times V_{P V}=3.50 \times 17.10=59.850$ W.

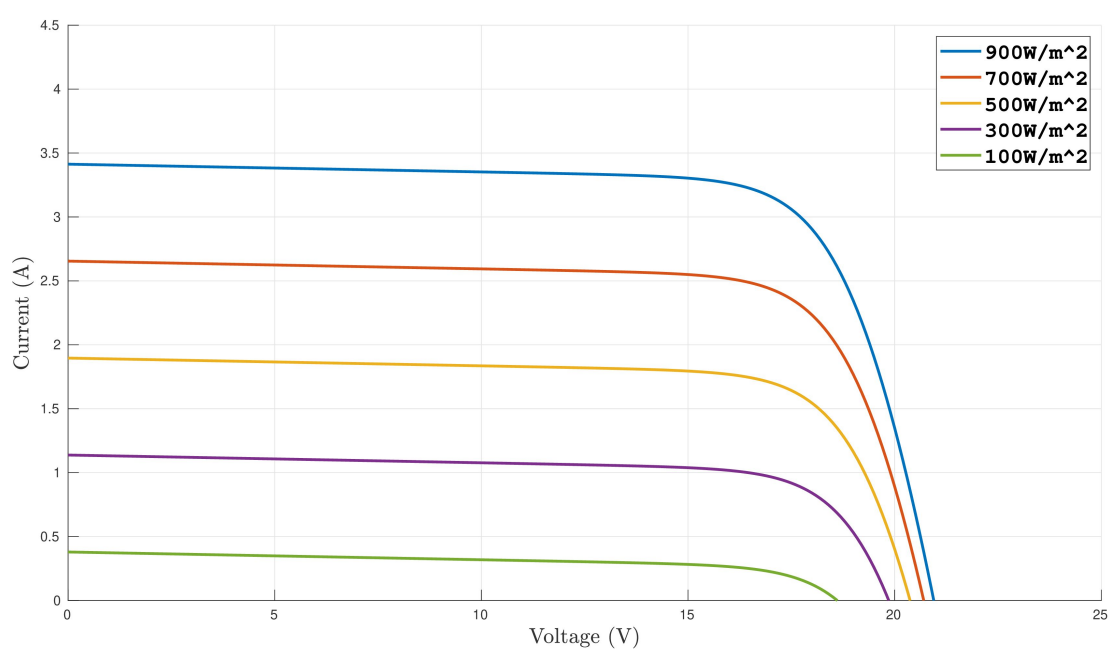

Figure 11: The Current-Voltage Characteristics Curve

To analyse the impact of meteorological data on the system characteristics (voltage, current and power), the I-V characteristic curves at various values of solar irradiance and temperature have been produced. The performance of the PV system is highly affected by the variability in the meteorological data. These are shown in the Figures 11 and 12.

It could be observed that the system current $\left(I_{P V}\right)$ increases quasilinearly as solar irradiance and the voltage $\left(V_{P V}\right)$ is in logarithmic pattern with irradiance. The maximum power increases faster than the solar irradiance and this implies that the PV efficiency is at its best especially at a higher level of irradiance. 


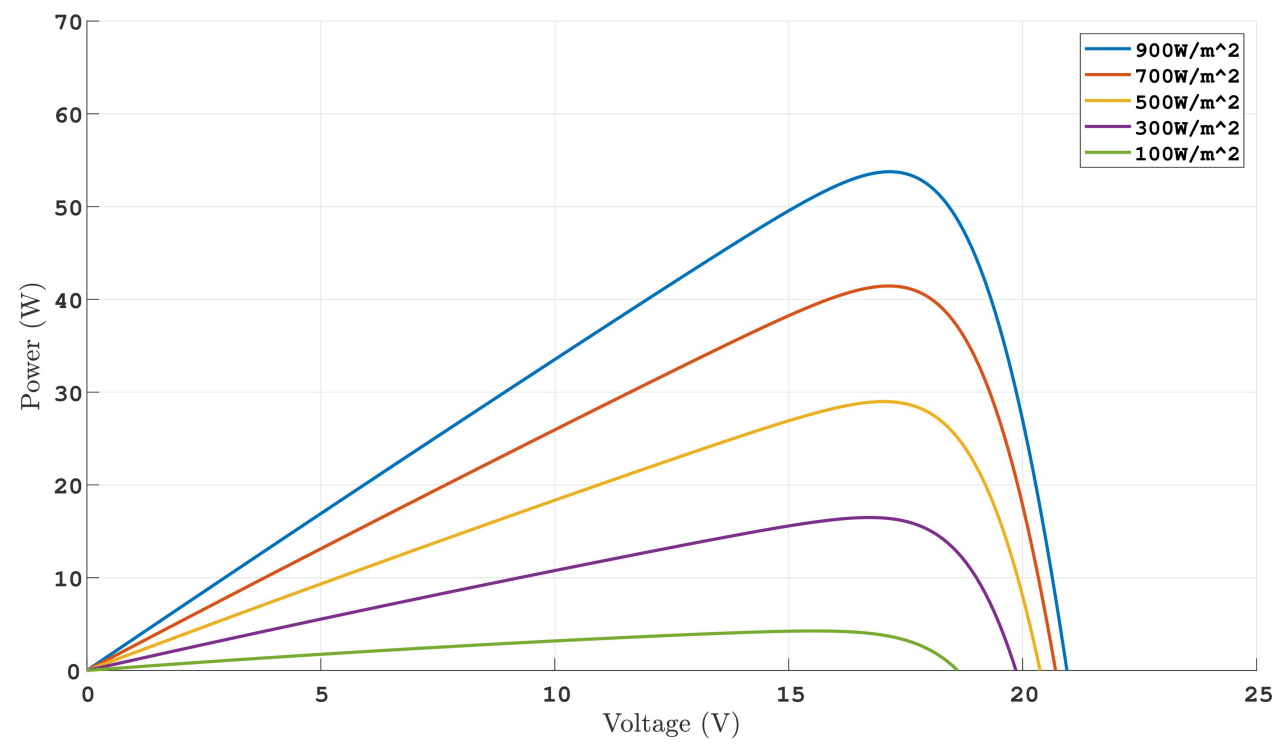

Figure 12: Irradiance effect on power.

Again, it could be observed that the temperature increment minimises the PV output voltage linearly and the PV module output current logarithmically and this is due to the better absorption of light (since the gap energy gets decreased with temperature). The peak PV power strongly decreases with temperature. These are shown in the Figures 13 and 14.

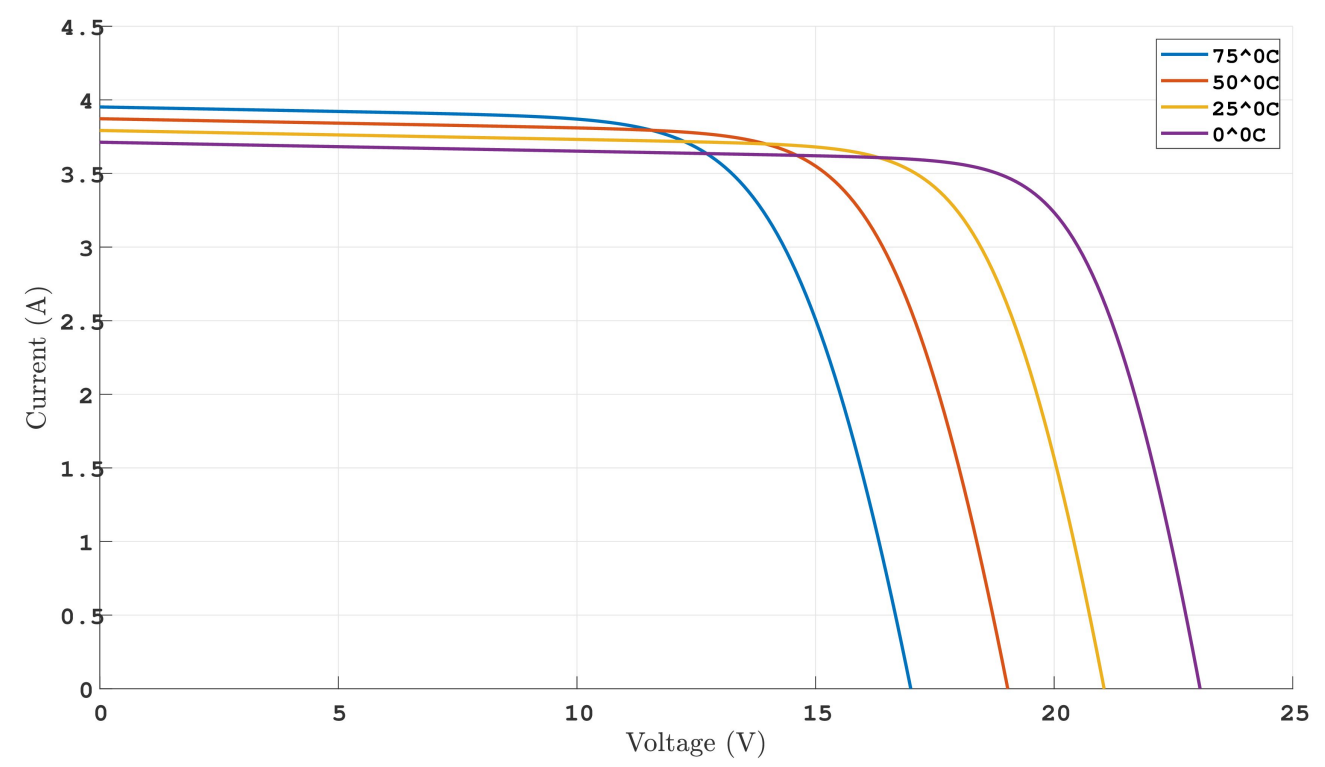

Figure 13: Temperature effect on Current and Voltage 


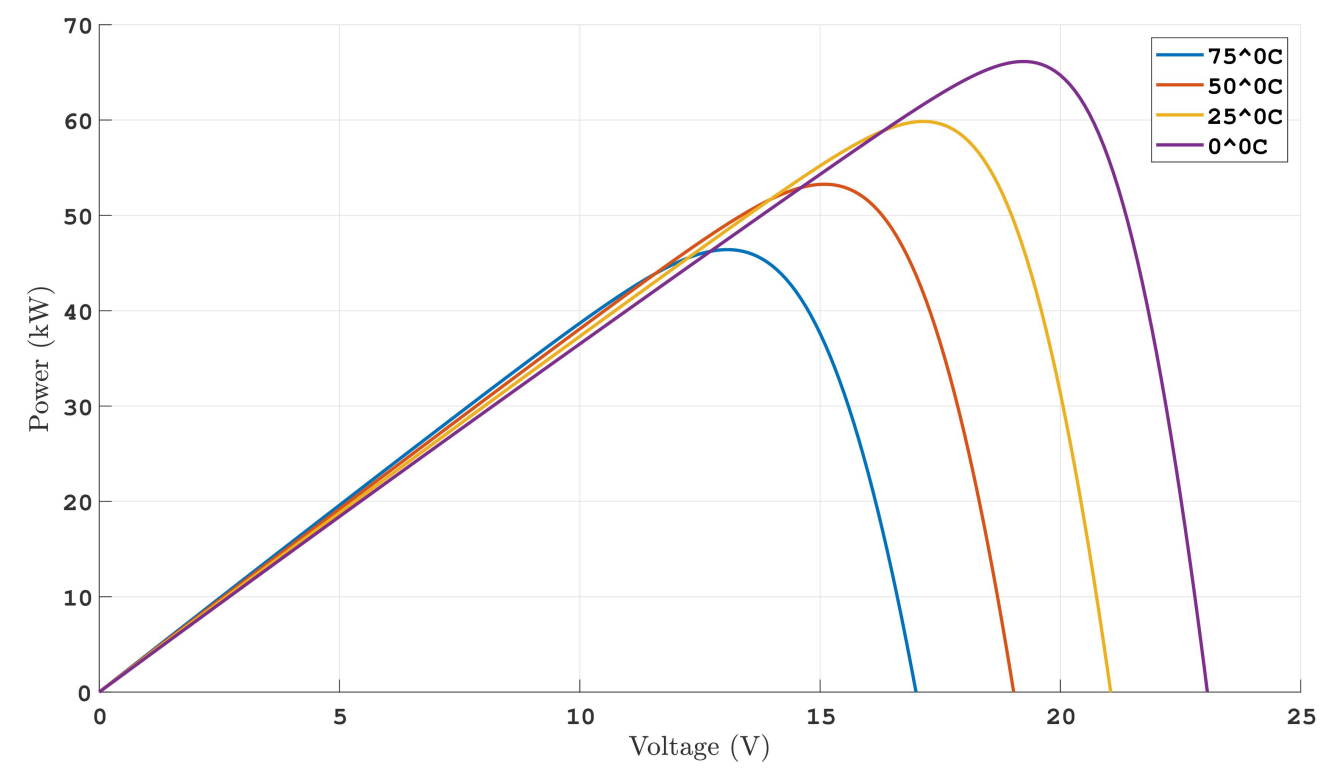

Figure 14: Temperature effect on the PV power.

\section{Conclusion and Recommendations}

1. In this study, different optimization algorithms have been applied to extract PV parameters. The PV cell model employed was the double diode model due to its high accuracy.

2. Sample Average Approximation was employed to handle to randomness effect in this problem. This makes the solution approach much more realistic than assuming predefined values of the solar irradiance and the temperature.

This implies that under fluctuating weather conditions, the model would be able to extract the most optimal Solar PV parameters to maximize power.

3. The algorithms were assessed based on the accuracy (higher power value) and a faster convergence speed. Among the methods, the HPSO-GWO produced the parameter $: R_{S}=$ 1.4623, $R_{s h}=215.0000, n_{1}=0.9500, n_{2}=1.6500$ with a maximum power delivered by a PV cell at current and voltage is given as $P_{t}=I_{P V} \times V_{P V}=3.50 \times 17.10=59.850 \mathrm{~W}$. Hence it could be concluded that the Hybrid PSO-GWO tends to find the most optimal solution at a relatively higher convergence rate. 
Academic Journal of Research and Scientific Publishing | Vol 3 | Issue 28

Publication Date: 5-8-2021 ISSN: 2706-6495

\section{Recommendations for Further Studies}

In this study a double diode model was used but using a three diode model should be considered in future studies as it could results in a higher accuracy though its complexity and computational strength would be higher.

\section{References}

Abbassi, R., Abbassi, A., Jemli, M., and Chebbi, S. (2018). Identification of unknown parameters of solar cell models: A comprehensive overview of available approaches. Renewable and Sustainable Energy Reviews, 90:453 474.

Birge, John R \& Louveaux, F. (2011). Introduction to stochastic programming.

Del Valle, Y., Venayagamoorthy, G. K., Mohagheghi, S., Hernandez, J.-C., and Harley, R. G. (2008). Particle swarm optimization: basic concepts, variants and applications in power systems. IEEE Transactions on evolutionary computation, 12(2):171-195.

Djamila (2018). Optimization of photovoltaic power systems modelization, simulation and control. Green Energy and Technology, 126(6)(3):929-935.

Elbaset, A. A., Ali, H., and Abd-El Sattar, M. (2015). Modeling of photovoltaic module based on two-diode model.

Gao, X., Cui, Y., Hu, J., Xu, G., Wang, Z., Qu, J., and Wang, H. (2018). Parameter extraction of solar cell models using improved shuffled complex evolution algorithm. Energy conversion and management, 157:460-479.

Ghaedi, A., Abbaspour, A., Fotuhi-Friuzabad, M., and Parvania, M. (2014). Incorporating large photovoltaic farms in power generation system adequacy assessment. Scientia Iranica, 21(3):924-934.

Hanifi, H., Khan, M. Z., Jaeckel, B., Hagendorf, C., Schneider, J., Abdallah, A., and Ilse, K. (2020). Optimum pv module interconnection layout and mounting orientation to reduce inhomogeneous soiling losses in desert environments. Solar Energy, 203:267-274. 
Ishaque, K., Salam, Z., and Taheri, H. (2011a). Simple, fast and accurate two-diode model for photovoltaic modules. Solar energy materials and solar cells, 95(2):586-594.

Ishaque, Kashif, S. Z., Taheri, H., et al. (2011b). Modeling and simulation of photovoltaic (pv) system during partial shading based on a two-diode model. Simulation Modelling Practice and Theory, 19(7):1613-1626.

Islam, Anek, C. I. (2014). Simulation of two diode model based pv solar cell/array: a simulink approach. In 1st International Conference on research in Science, Engineering\& Management (IOCRSEM 2014).

Ji, Wu \& Chee, K. C. (2011). Prediction of hourly solar radiation using a novel hybrid model of arma and tdnn. Solar Energy, 85(5):808-817.

Kamjoo, A. (2015). A Decision Support System for Integrated Design of Hybrid Renewable Energy System. PhD thesis, Northumbria University.

Lim, L. H. I., Ye, Z., Ye, J., Yang, D., and Du, H. (2015). A linear method to extract diode model parameters of solar panels from a single i-v curve. Renewable Energy, 76:135-142.

Liu, Z. and Zhang, Y. (2018). Optimal planning and operation of photovoltaic-storage systems based on multi-period voltage control at pcc. In 2018 International Conference on Power System Technology (POWERCON), pages 1372-1377. IEEE.

Magazine, P. (2020). Solar to play key role in ghana's us 230 million renewable energy program.

Miao, D., Chen, W., Zhao, W., and Demsas, T. (2020). Parameter estimation of pem fuel cells employing the hybrid grey wolf optimization method. Energy, 193:116616.

Mirjalili, S., Mirjalili, S. M., and Lewis, A. (2014). Grey wolf optimizer. Advances in engineering software, 69:46-61.

Nguyen, Xuan Hieu, M. P. (2015). Mathematical modeling of photovoltaic cell/module/arrays with tags in matlab/simulink. Environmental Systems Research, 4(1):24. 
Academic Journal of Research and Scientific Publishing | Vol 3 | Issue 28

Publication Date: 5-8-2021 ISSN: 2706-6495

Parizad, A., Khazali, A., and Kalantar, M. (2010). Optimal placement of distributed generation with sensitivity factors considering voltage stability and losses indices. In 2010 18th Iranian Conference on Electrical Engineering, pages 848-855. IEEE.

R. Shah, N. Mithulananathan, R. B. K. Y. L. . A. L. (2017). Influence of large-scale pv on voltage stability of sub-transmission system. Int. J. Electr. Eng. Inf., 11(11):148-161.

Senel, F. A., Gok,ce, F., Yuksel, A. S., and Yigit, T. (2019). A novel hybrid pso-gwo algorithm for optimization problems. Engineering with Computers, 35(4):1359-1373.

Sharafi, Masoud \& ELMekkawy, T. Y. (2014). Multi-objective optimal design of hybrid renewable energy systems using pso-simulation based approach. Renewable Energy, 68:6779.

Sunpower (2020). Sun power: an introduction to the applications of solar energy.

Tian, Dongping, S. Z. (2018). Mpso: Modified particle swarm optimization and its applications. Swarm and evolution.

Copyright (C) 2021 Ali Abubakar, Dr. Reindorf Nartey Borkor, AJRSP. This is an open-access article distributed under the terms of the Creative Commons Attribution License (CC BY NC).

Doi: doi.org/10.52132/Ajrsp.e.2021.281 